\title{
BIFURCATION OF POSITIVE PERIODIC SOLUTIONS TO NON-AUTONOMOUS UNDAMPED DUFFING EQUATIONS
}

\author{
JIŘÍ ŠREMR
}

\begin{abstract}
We study a bifurcation of positive solutions to the parameter-dependent periodic problem

$$
u^{\prime \prime}=p(t) u-h(t)|u|^{\lambda} \operatorname{sgn} u+\mu f(t) ; \quad u(0)=u(\omega), u^{\prime}(0)=u^{\prime}(\omega),
$$

where $\lambda>1, p, h, f \in L([0, \omega])$, and $\mu \in \mathbb{R}$ is a parameter. Both the coefficient $p$ and the forcing term $f$ may change their signs, $h \geq 0$ a. e. on $[0, \omega]$. We provide sharp conditions on the existence and multiplicity as well as non-existence of positive solutions to the given problem depending on the choice of the parameter $\mu$.
\end{abstract}

\section{INTRODUCTION}

Consider the parameter-dependent problem

$$
u^{\prime \prime}=p(t) u-h(t)|u|^{\lambda} \operatorname{sgn} u+\mu f(t) ; \quad u(0)=u(\omega), u^{\prime}(0)=u^{\prime}(\omega),
$$

where $p, h, f \in L([0, \omega]), h \geq 0$ a.e. on $[0, \omega], \lambda>1$, and $\mu \in \mathbb{R}$ is a parameter. By a solution to problem (1.1), as usual, we understand a function $u:[0, \omega] \rightarrow \mathbb{R}$ which is absolutely continuous together with its first derivative, satisfies the given equation almost everywhere, and meets the periodic conditions.

We first note that the differential equation in (1.1) with $\lambda=3$ is derived, for example, when approximating a non-linearity in the equation of motion of the oscillator illustrated in Fig. 1. Consider a forced undamped oscillator consisting of a mass body of weight $m$ and a linear spring of characteristic $k$ and non-deformed length $\ell$. Assume that the mass body moves horizontally without any friction and the spring's base point $B$ oscillates vertically, i.e., $d$ is a positive $\omega$-periodic function. This is a system with a single degree of freedom, described by the coordinate $x$, whose equation of motion is of the form

$$
x^{\prime \prime}=\frac{k}{m} x\left(\frac{\ell}{\sqrt{d^{2}(t)+x^{2}}}-1\right)+\frac{F(t)}{m} .
$$

A classical approach to deriving Duffing equation is to approximate the nonlinearity in (1.2) by a third-order Taylor polynomial centred at 0 . We thus get the

$M S C$ (2010): primary 34B08; secondary 34C23, 34C25, 34B18.

Keywords: periodic solution, second-order differential equation, Duffing equation, existence, multiplicity, bifurcation, positive solution.

The research has been supported by the internal grant FSI-S-20-6187 of FME BUT. 


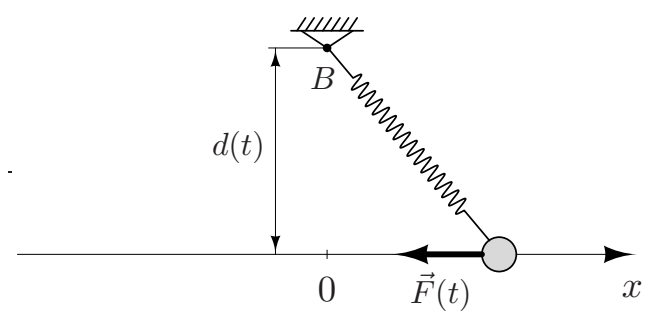

Figure 1. Forced undamped oscillator.

equation

$$
x^{\prime \prime}=\frac{k(\ell-d(t))}{m d(t)} x-\frac{k \ell}{2 m d^{3}(t)} x^{3}+\frac{F(t)}{m},
$$

which is a particular case of the differential equation in (1.1). It is worth mentioning that the results of the present paper can be applied, for instance, to the forcing terms

$$
F(t):=-f_{0}, \quad F(t):=A\left(\sin \frac{2 \pi t}{\omega}-\frac{1}{2}\right),
$$

where $f_{0}, A>0$. Hence, Theorem 3.1 below provides information about the exact multiplicity of positive $\omega$-periodic solutions to equation (1.3) depending on the value of $f_{0}$, resp. $A$ (for discussion, see Section 6 ).

For the results covering the multiplicity and local/global bifurcations of periodic solutions to Duffing equations, we refer readers, for instance, to $[2,4,5,8]$ (see also references therein). In $[2,4,8]$, the authors study the parameter-dependent problems for second-order differential equations assuming a strong damped condition and a sign-constant forcing term. In the present paper, we consider an undamped non-autonomous Duffing equation with a linear part of the class $\mathcal{V}^{-}(\omega)$ (see Definition 2.1, Remark 3.2) and a forcing term $f$, which may change its sign. We use the results presented in [9] and show the existence and multiplicity as well as non-existence of positive solutions to problem (1.1) depending on the choice of the parameter $\mu$.

Let us show, as a motivation, what happens in the autonomous case of (1.1). Hence, consider the equation

$$
x^{\prime \prime}=a x-b|x|^{\lambda} \operatorname{sgn} x-\mu,
$$

where $a, b>0$ and $\mu \in \mathbb{R}$. By direct calculation, the phase portraits of this equation can be elaborated depending on the choice of the parameter $\mu$ and, thus, one can prove the following proposition concerning the positive periodic solutions to equation (1.5).

Proposition 1.1. Let $\lambda>1$ and $a, b>0$. Then, the following conclusions hold:

(i) If $\mu \leq 0$, then equation (1.5) has a unique positive equilibrium (center) and non-constant positive periodic solutions with different periods.

(ii) If $0<\mu<\frac{(\lambda-1) a}{\lambda}\left(\frac{a}{\lambda b}\right)^{\frac{1}{\lambda-1}}$, then equation (1.5) possesses exactly two positive equilibria $x_{2}>x_{1}\left(x_{1}\right.$ is a saddle and $x_{2}$ is a center) and non-constant 
positive periodic solutions with different periods. Moreover, all non-constant positive periodic solutions are greater than $x_{1}$ and oscillate around $x_{2}$.

(iii) If $\mu=\frac{(\lambda-1) a}{\lambda}\left(\frac{a}{\lambda b}\right)^{\frac{1}{\lambda-1}}$, then equation (1.5) has a unique positive equilibrium (cusp) and no non-constant positive periodic solution occurs.

(iv) If $\mu>\frac{(\lambda-1) a}{\lambda}\left(\frac{a}{\lambda b}\right)^{\frac{1}{\lambda-1}}$, then equation (1.5) has no positive periodic solution.

Proposition 1.1 shows that, if we consider $\mu$ as a bifurcation parameter, then, crossing the value $\frac{(\lambda-1) a}{\lambda}\left(\frac{a}{\lambda b}\right)^{\frac{1}{\lambda-1}}$, a bifurcation of positive periodic solutions to equation (1.5) occurs. In Section 3, we extend conclusions (ii)-(iv) of Proposition 1.1 for the non-autonomous problem (1.1) with the forcing term $f$ satisfying $\int_{0}^{\omega} f(s) \mathrm{d} s<0$.

\section{Notation AND DEFinitons}

The following notation is used throughout the paper:

- $\mathbb{R}$ is the set of real numbers. For $x \in \mathbb{R}$, we put $[x]_{+}=\frac{1}{2}(|x|+x)$ and $[x]_{-}=$ $\frac{1}{2}(|x|-x)$.

- $C(I)$ denotes the set of continuous real functions defined on the interval $I \subseteq \mathbb{R}$. For $u \in C([a, b])$, we put $\|u\|_{C}=\max \{|u(t)|: t \in[a, b]\}$.

- $A C^{1}([a, b])$ is the set of functions $u:[a, b] \rightarrow \mathbb{R}$ which are absolutely continuous together with their first derivatives.

- $A C_{\ell}([a, b])$ (resp. $\left.A C_{u}([a, b])\right)$ is the set of absolutely continuous functions $u:[a, b] \rightarrow \mathbb{R}$ such that $u^{\prime}$ admits the representation $u^{\prime}(t)=\gamma(t)+\sigma(t)$ for a.e. $t \in[a, b]$, where $\gamma:[a, b] \rightarrow \mathbb{R}$ is absolutely continuous and $\sigma:[a, b] \rightarrow \mathbb{R}$ is a non-decreasing (resp. non-increasing) function whose derivative is equal to zero almost everywhere on $[a, b]$.

- $L([a, b])$ is the Banach space of Lebesgue integrable functions $p:[a, b] \rightarrow \mathbb{R}$ equipped with the norm $\|p\|_{L}=\int_{a}^{b}|p(s)| \mathrm{d} s$. The symbol Int $A$ stands for the interior of the set $A \subset L([a, b])$.

Definition 2.1. ([6, Definitions 0.1 and 15.1, Proposition 15.2]) We say that a function $p \in L([0, \omega])$ belongs to the set $\mathcal{V}^{-}(\omega)$ if, for any function $u \in A C^{1}([0, \omega])$ satisfying

$$
u^{\prime \prime}(t) \geq p(t) u(t) \quad \text { for a.e. } t \in[0, \omega], \quad u(0)=u(\omega), \quad u^{\prime}(0) \geq u^{\prime}(\omega),
$$

the inequality $u(t) \leq 0$ holds for $t \in[0, \omega]$.

Remark 2.2. Let $\omega>0$. If $p(t):=p_{0}$ for $t \in[0, \omega]$, then one can show by direct calculation that $p \in \mathcal{V}^{-}(\omega)$ if and only if $p_{0}>0$. For non-constant functions $p \in L([0, \omega])$, efficient conditions guaranteeing the inclusion $p \in \mathcal{V}^{-}(\omega)$ are provided in $[6]$ (see also $[1,10])$.

Remark 2.3. It is well known that, if the homogeneous problem

$$
u^{\prime \prime}=p(t) u ; \quad u(0)=u(\omega), u^{\prime}(0)=u^{\prime}(\omega)
$$

has only the trivial solution, then, for any $f \in L([0, \omega])$, the problem

$$
u^{\prime \prime}=p(t) u+f(t) ; \quad u(0)=u(\omega), u^{\prime}(0)=u^{\prime}(\omega)
$$


possesses a unique solution $u$ and this solution satisfies

$$
|u(t)| \leq \Delta(p) \int_{0}^{\omega}|f(s)| \mathrm{d} s \quad \text { for } t \in[0, \omega],
$$

where $\Delta(p)$, depending only on $p$, denotes a norm of the Green's operator of problem (2.1). Clearly, $\Delta(p)>0$.

Remark 2.4. If $p \in \mathcal{V}^{-}(\omega)$, then problem (2.1) has only the trivial solution and the number $\Delta(p)$ defined in Remark 2.3 can be estimated, for example, by using a minimal value of the Green's function of problem (2.1) (see, e. g., [10]).

For instance, if $p(t):=p_{0}$ for $t \in[0, \omega]$ and $p_{0}>0$, then

$$
\Delta(p) \leq\left(2 \sqrt{p_{0}} \tanh \frac{\omega \sqrt{p_{0}}}{2}\right)^{-1}<\left(\frac{\omega p_{0}}{\cosh \frac{\omega \sqrt{p_{0}}}{2}}\right)^{-1} .
$$

Definition 2.5 ([6, Definition 16.1]). Let $p, f \in L([0, \omega])$. We say that the pair $(p, f)$ belongs to the set $\mathcal{U}(\omega)$ if problem $(2.2)$ has a unique solution which is positive.

\section{Main Results}

Theorem 3.1. Let $\lambda>1, p \in \mathcal{V}^{-}(\omega)$, and

$$
\begin{gathered}
h(t) \geq 0 \quad \text { for a. e. } t \in[0, \omega], \quad h(t) \not \equiv 0, \\
(p, f) \in \mathcal{U}(\omega), \quad \int_{0}^{\omega} f(s) \mathrm{d} s<0 .
\end{gathered}
$$

Then, there exists $\left.\mu_{0} \in\right] 0,+\infty[$ such that the following conclusions hold:

(1) If $\mu=0$, then problem (1.1) has at least one positive solution and, for any couple of distinct positive solutions $u_{1}, u_{2}$ to (1.1), the conditions

$$
\begin{aligned}
& \min \left\{u_{1}(t)-u_{2}(t): t \in[0, \omega]\right\}<0, \\
& \max \left\{u_{1}(t)-u_{2}(t): t \in[0, \omega]\right\}>0
\end{aligned}
$$

hold. If, moreover,

$$
\mathrm{e}^{-1+\sqrt{1+\omega \int_{0}^{\omega} p(s) \mathrm{d} s}}\left(-1+\sqrt{1+\omega \int_{0}^{\omega} p(s) \mathrm{d} s}\right) \leq 8 \lambda^{*},
$$

where

$$
\lambda^{*}:= \begin{cases}\left\lfloor\frac{1}{\lambda-1}\right\rfloor & \text { for } \lambda \in] 1,2] \\ \frac{1}{\lceil\lambda-1\rceil} & \text { for } \lambda>2\end{cases}
$$

in which $\lfloor\cdot\rfloor$ and $\lceil\cdot\rceil$ denote the floor function and ceiling function, respectively, then problem (1.1) with $\mu=0$ has a unique positive solution.

(2) If $0<\mu<\mu_{0}$, then problem (1.1) has solutions $u_{1}$, $u_{2}$ such that

$$
u_{2}(t)>u_{1}(t)>0 \quad \text { for } t \in[0, \omega]
$$

and, for any non-negative solution u to problem (1.1) satisfying

$$
u(t) \not \equiv u_{1}(t), \quad u(t) \not \equiv u_{2}(t),
$$


the conditions

$$
u(t)>u_{1}(t) \quad \text { for } t \in[0, \omega]
$$

and

$$
\begin{aligned}
& \min \left\{u(t)-u_{2}(t): t \in[0, \omega]\right\}<0, \\
& \max \left\{u(t)-u_{2}(t): t \in[0, \omega]\right\}>0
\end{aligned}
$$

hold.

(3) If $\mu=\mu_{0}$, then problem (1.1) has a unique positive solution.

(4) If $\mu>\mu_{0}$, then problem (1.1) has no positive solution.

Open questions. The following two questions remain open in Theorem 3.1:

(1) Does there exist, for any $\omega>0$, a positive solution $u$ to problem (1.1) satisfying (3.5) in conclusion (2)?

(2) What happens in the case of $\mu<0$ ?

Remark 3.2. By virtue of $\left[6\right.$, Theorem 11.1], the hypothesis $p \in \mathcal{V}^{-}(\omega)$ of Theorem 3.1 is satisfied, for instance, if one of the following conditions hold:

(a)

(b)

$$
p(t) \geq 0 \quad \text { for a. e. } t \in[0, \omega], \quad p(t) \not \equiv 0,
$$

$$
0<\int_{0}^{\omega}[p(s)]_{-} \mathrm{d} s<\frac{4}{\omega}, \quad \int_{0}^{\omega}[p(s)]_{+} \mathrm{d} s \geq \frac{\int_{0}^{\omega}[p(s)]_{-} \mathrm{d} s}{1-\frac{\omega}{4} \int_{0}^{\omega}[p(s)]_{-} \mathrm{d} s} .
$$

Other efficient conditions guaranteeing the inclusion $p \in \mathcal{V}^{-}(\omega)$ and their consequences for particular cases of the coefficient $p$ are available in [6].

Remark 3.3. Let $p \in \mathcal{V}^{-}(\omega)$. It follows from [6, Theorem 16.2] that hypothesis (3.2) of Theorem 3.1 holds, provided that

$$
\int_{0}^{\omega}[f(s)]_{-} \mathrm{d} s>\mathrm{e}^{\frac{\omega}{4} \int_{0}^{\omega}[p(s)]_{+} \mathrm{d} s} \int_{0}^{\omega}[f(s)]_{+} \mathrm{d} s .
$$

In particular, if

$$
f(t) \leq 0 \quad \text { for a. e. } t \in[0, \omega], \quad f(t) \not \equiv 0,
$$

then (3.2) is satisfied.

We now provide lower and upper estimates of the number $\mu_{0}$ appearing in the conclusion of Theorem 3.1.

Proposition 3.4. Let $\lambda>1, p \in \mathcal{V}^{-}(\omega)$, h satify (3.1), and $f$ be such that (3.8) holds. Then, the number $\mu_{0}$ appearing in the conclusion of Theorem 3.1 satisfies

$$
\mu_{0} \geq \frac{(\lambda-1)[\Delta(p)]^{-\frac{\lambda}{\lambda-1}}}{\lambda\left[\lambda \int_{0}^{\omega} h(s) \mathrm{d} s\right]^{\frac{1}{\lambda-1}} \int_{0}^{\omega}[f(s)]_{-} \mathrm{d} s},
$$


where $\Delta$ is defined in Remark 2.3, and

$$
\mu_{0}<\frac{(\lambda-1)\left[\mathrm{e}^{\frac{\omega}{4} \int_{0}^{\omega}[p(s)]_{+} \mathrm{d} s} \int_{0}^{\omega}[p(s)]_{+} \mathrm{d} s-\int_{0}^{\omega}[p(s)]_{-} \mathrm{d} s\right]^{\frac{\lambda}{\lambda-1}}}{\lambda\left[\lambda \int_{0}^{\omega} h(s) \mathrm{d} s\right]^{\frac{1}{\lambda-1}}\left[\int_{0}^{\omega}[f(s)]_{-} \mathrm{d} s-\mathrm{e}^{\frac{\omega}{4} \int_{0}^{\omega}[p(s)]_{+} \mathrm{d} s} \int_{0}^{\omega}[f(s)]_{+} \mathrm{d} s\right]} .
$$

Remark 3.5. If $p \in \mathcal{V}^{-}(\omega)$, then [6, Proposition 10.8] yields $\int_{0}^{\omega} p(s) \mathrm{d} s>0$. Therefore, inequality (3.11) in Proposition 3.4 is consistent.

Remark 3.6. Theorem 3.1 extends conclusions ((ii))-((iv)) of Proposition 1.1 for the non-autonomous Duffing equations with a sign-changing forcing term. Indeed, let $\omega>0$ and

$$
p(t):=a, \quad h(t):=b, \quad f(t):=-1 \quad \text { for } t \in[0, \omega],
$$

where $a, b>0$. Then, $p \in \mathcal{V}^{-}(\omega)$ (see Remark 2.2), $h$ and $f$ satisfy (3.1) and (3.9), respectively, and conclusions $((2))-((4))$ of Theorem 3.1 coincide with those in Proposition 1.1. Moreover, the number $\Delta(p)$ satisfies (2.3) and, thus, the number $\mu_{0}$ appearing in Proposition 3.4 satisfies

$$
\left(\frac{1}{\cosh \frac{\omega \sqrt{a}}{2}}\right)^{\frac{\lambda}{\lambda-1}} \frac{(\lambda-1) a}{\lambda}\left(\frac{a}{\lambda b}\right)^{\frac{1}{\lambda-1}}<\mu_{0}<\left(\mathrm{e}^{\frac{\omega^{2} a}{4}}\right)^{\frac{\lambda}{\lambda-1}} \frac{(\lambda-1) a}{\lambda}\left(\frac{a}{\lambda b}\right)^{\frac{1}{\lambda-1}}
$$

compare it with the number appearing in Proposition 1.1.

\section{Auxiliary statements}

We first recall some results stated in [9].

Lemma 4.1 ([9, Theorem 3.6]). Let $\lambda>1, \mu \in \mathbb{R}, p \in \mathcal{V}^{-}(\omega),(p, \mu f) \in \mathcal{U}(\omega)$, and $h$ satisfy (3.1). Let, moreover, there exist a positive function $\beta \in A C_{u}([0, \omega])$ such that

$$
\begin{gathered}
\beta^{\prime \prime}(t) \leq p(t) \beta(t)-h(t) \beta^{\lambda}(t)+\mu f(t) \quad \text { for a. e. } t \in[0, \omega], \\
\beta(0)=\beta(\omega), \quad \beta^{\prime}(0)=\beta^{\prime}(\omega) .
\end{gathered}
$$

Then, problem (1.1) has a positive solution $u_{*}$ such that every non-negative solution u to problem (1.1) satisfies

$$
\text { either } u(t)>u_{*}(t) \quad \text { for } t \in[0, \omega], \quad \text { or } \quad u(t) \equiv u_{*}(t) \text {. }
$$

Moreover, for any couple of distinct positive solutions $u_{1}, u_{2}$ to (1.1) satisfying

$$
u_{1}(t) \not \equiv u_{*}(t), \quad u_{2}(t) \not \equiv u_{*}(t),
$$

conditions (3.3) hold.

Lemma $4.2\left(\left[9\right.\right.$, Theorem 3.7]). Let $\lambda>1, \mu \in \mathbb{R}, p \in \mathcal{V}^{-}(\omega),(p, \mu f) \in \mathcal{U}(\omega)$, and $h$ satisfy (3.1). Let, moreover, there exist functions $\beta_{1} \in A C^{1}([0, \omega])$ and $\beta_{2} \in A C_{u}([0, \omega])$ such that

$$
\begin{gathered}
0<\beta_{1}(t)<\beta_{2}(t) \quad \text { for } t \in[0, \omega] \\
\beta_{k}^{\prime \prime}(t) \leq p(t) \beta_{k}(t)-h(t) \beta_{k}^{\lambda}(t)+\mu f(t) \quad \text { for a. e. } t \in[0, \omega], \quad k=1,2,
\end{gathered}
$$




$$
\beta_{k}(0)=\beta_{k}(\omega), \quad \beta_{k}^{\prime}(0)=\beta_{k}^{\prime}(\omega), \quad k=1,2 .
$$

Then, there exist solutions $u_{1}, u_{2}$ to problem (1.1) such that (3.4) is fulfilled and, for any non-negative solution u to problem (1.1) satisfying (3.5), conditions (3.6) and (3.7) hold.

Lemma $4.3\left(\left[9\right.\right.$, Corollary 3.9(ii)]). Let $\lambda>1, \mu \in \mathbb{R}, p \in \mathcal{V}^{-}(\omega),(p, \mu f) \in$ $\mathcal{U}(\omega)$, and $h$ satisfy $(3.1)$. If

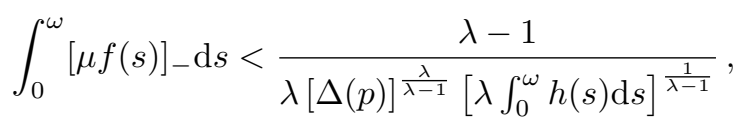

where $\Delta$ is defined in Remark 2.3, then the conclusion of Lemma 4.2 holds.

Lemma 4.4 ([9, Theorem 3.11]). Let $\lambda>1, \mu \in \mathbb{R} \backslash\{0\}, p \in \mathcal{V}^{-}(\omega)$, h satisfy (3.1), and

$$
\begin{aligned}
& \int_{0}^{\omega}[\mu f(s)]_{-} \mathrm{d} s-\mathrm{e}^{\frac{\omega}{4} \int_{0}^{\omega}[p(s)]_{+} \mathrm{d} s} \int_{0}^{\omega}[\mu f(s)]_{+} \mathrm{d} s \\
& \geq \frac{\lambda-1}{\lambda} \frac{\left[\mathrm{e}^{\frac{\omega}{4} \int_{0}^{\omega}[p(s)]_{+} \mathrm{d} s} \int_{0}^{\omega}[p(s)]_{+} \mathrm{d} s-\int_{0}^{\omega}[p(s)]_{-} \mathrm{d} s\right]^{\frac{\lambda}{\lambda-1}}}{\left[\lambda \int_{0}^{\omega} h(s) \mathrm{d} s\right]^{\frac{1}{\lambda-1}}} .
\end{aligned}
$$

Then, problem (1.1) has no non-negative solution.

Lemma 4.5 ([6, Theorem 16.2]). Let $p \in \mathcal{V}^{-}(\omega)$. Then, there exists $\nu>0$ such that, for any non-positive function $q \in L([0, \omega])$, the problem

$$
z^{\prime \prime}=p(t) z+q(t) ; \quad z(0)=z(\omega), z^{\prime}(0)=z^{\prime}(\omega)
$$

has a unique solution $z$ and this solution satisfies

$$
z(t) \geq \nu \int_{0}^{\omega}|q(s)| \mathrm{d} s \quad \text { for } t \in[0, \omega] .
$$

Lemma 4.6. Let $\lambda>1$, conditions (3.1) and (3.2) hold, $\left\{\mu_{n}\right\}_{n=1}^{\infty}$ be a sequence of positive numbers and let, for any $n \in \mathbb{N}, u_{n}$ be a positive solution to problem (1.1) with $\mu=\mu_{n}$. Then, the sequences $\left\{\left\|u_{n}\right\|_{C}\right\}_{n=1}^{\infty}$ and $\left\{\mu_{n}\right\}_{n=1}^{\infty}$ are bounded.

Proof. We first show that

$$
\sup \left\{\left\|u_{n}\right\|_{C}: n \in \mathbb{N}\right\}<+\infty .
$$

Suppose on the contrary that (4.7) does not hold. Then, we can assume without loss of generality that

$$
\lim _{n \rightarrow+\infty}\left\|u_{n}\right\|_{C}=+\infty
$$

Put

Clearly,

$$
v_{n}(t):=\frac{u_{n}(t)}{\left\|u_{n}\right\|_{C}} \quad \text { for } t \in[0, \omega], n \in \mathbb{N} .
$$

$$
\left\|v_{n}\right\|_{C}=1, \quad v_{n}(t)>0 \quad \text { for } t \in[0, \omega], n \in \mathbb{N} .
$$

It follows from (1.1) with $\mu=\mu_{n}$ that, for any $n \in \mathbb{N}$,

$$
v_{n}^{\prime \prime}(t)=p(t) v_{n}(t)-\left\|u_{n}\right\|_{C}^{\lambda-1} h(t) v_{n}^{\lambda}(t)+\frac{\mu_{n}}{\left\|u_{n}\right\|_{C}} f(t) \quad \text { for a. e. } t \in[0, \omega]
$$


which yields

$$
0=\int_{0}^{\omega} p(s) v_{n}(s) \mathrm{d} s-\left\|u_{n}\right\|_{C}^{\lambda-1} \int_{0}^{\omega} h(s) v_{n}^{\lambda}(s) \mathrm{d} s+\frac{\mu_{n}}{\left\|u_{n}\right\|_{C}} \int_{0}^{\omega} f(s) \mathrm{d} s
$$

for $n \in \mathbb{N}$. In view of (3.2) and (4.9), from the latter equality, we get

$$
\left\|u_{n}\right\|_{C}^{\lambda-1} \int_{0}^{\omega} h(s) v_{n}^{\lambda}(s) \mathrm{d} s+\frac{\mu_{n}}{\left\|u_{n}\right\|_{C}}\left|\int_{0}^{\omega} f(s) \mathrm{d} s\right| \leq \int_{0}^{\omega}|p(s)| \mathrm{d} s \quad \text { for } n \in \mathbb{N} .
$$

Put

$$
A:=\sup \left\{\left\|u_{n}\right\|_{C}^{\lambda-1} \int_{0}^{\omega} h(s) v_{n}^{\lambda}(s) \mathrm{d} s: n \in \mathbb{N}\right\}, \quad B:=\sup \left\{\frac{\mu_{n}}{\left\|u_{n}\right\|_{C}}: n \in \mathbb{N}\right\} .
$$

By virtue of (3.1), (3.2) and (4.9), it follows from (4.11) that $A \in] 0,+\infty[, B \in$ ] $0,+\infty[$, and we can assume without loss of generality that

$$
\lim _{n \rightarrow+\infty}\left\|u_{n}\right\|_{C}^{\lambda-1} \int_{0}^{\omega} h(s) v_{n}^{\lambda}(s) \mathrm{d} s=h_{0}, \quad \lim _{n \rightarrow+\infty} \frac{\mu_{n}}{\left\|u_{n}\right\|_{C}}=\mu_{0},
$$

where

$$
h_{0} \geq 0, \quad \mu_{0} \geq 0 .
$$

For any $n \in \mathbb{N}$, we choose $t_{n} \in[0, \omega]$ such that $v_{n}^{\prime}\left(t_{n}\right)=0$. In view of $(3.1),(4.9)$, and (4.12), integrating (4.10) from $t_{n}$ to $t$, we get

$$
\begin{aligned}
\left|v_{n}^{\prime}(t)\right| & =\left|\int_{t_{n}}^{t}\left[p(s) v_{n}(s)-\left\|u_{n}\right\|_{C}^{\lambda-1} h(s) v_{n}^{\lambda}(s)+\frac{\mu_{n}}{\left\|u_{n}\right\|_{C}} f(s)\right] \mathrm{d} s\right| \\
& \leq \int_{0}^{\omega}|p(s)| \mathrm{d} s+\left\|u_{n}\right\|_{C}^{\lambda-1} \int_{0}^{\omega} h(s) v_{n}^{\lambda}(s) \mathrm{d} s+\frac{\mu_{n}}{\left\|u_{n}\right\|_{C}} \int_{0}^{\omega}|f(s)| \mathrm{d} s \\
& \leq \int_{0}^{\omega}|p(s)| \mathrm{d} s+A+B \int_{0}^{\omega}|f(s)| \mathrm{d} s \quad \text { for } t \in[0, \omega], n \in \mathbb{N} .
\end{aligned}
$$

Therefore, the sequences $\left\{\left\|v_{n}\right\|_{C}\right\}_{n=1}^{\infty}$ and $\left\{\left\|v_{n}^{\prime}\right\|_{C}\right\}_{n=1}^{\infty}$ are bounded and, thus, by the Arzelà-Ascoli theorem, we can assume without loss of generality that

$$
\lim _{n \rightarrow+\infty} v_{n}(t)=v_{0}(t) \quad \text { uniformly on }[0, \omega],
$$

where $v_{0} \in C([0, \omega])$. From (4.9), we get

$$
v_{0}(t) \geq 0 \quad \text { for } t \in[0, \omega], \quad\left\|v_{0}\right\|_{C}=1 .
$$

It follows from the hypothesis $(p, f) \in \mathcal{U}(\omega)$ that the problem

$$
v^{\prime \prime}=p(t) v+f(t) ; \quad v(0)=v(\omega), v^{\prime}(0)=v^{\prime}(\omega)
$$

has a unique solution $v$ which is positive. According to Lemma 4.5 (with $q(t):=$ $\left.-\left\|u_{n}\right\|_{C}^{\lambda-1} h(t) v_{n}^{\lambda}(t)\right)$, there exists $\nu>0$ such that, for any $n \in \mathbb{N}$, the problem

$$
w^{\prime \prime}=p(t) w-\left\|u_{n}\right\|_{C}^{\lambda-1} h(t) v_{n}^{\lambda}(t) ; \quad w(0)=w(\omega), w^{\prime}(0)=w^{\prime}(\omega)
$$

has a unique solution $w_{n}$ and

$$
w_{n}(t) \geq \nu\left\|u_{n}\right\|_{C}^{\lambda-1} \int_{0}^{\omega} h(s) v_{n}^{\lambda}(s) \mathrm{d} s \quad \text { for } t \in[0, \omega], n \in \mathbb{N} .
$$


It is clear that $v_{n}=w_{n}+\frac{\mu_{n}}{\left\|u_{n}\right\|_{C}} v$ for $n \in \mathbb{N}$. Therefore, (4.17) yields

$$
v_{n}(t) \geq \nu\left\|u_{n}\right\|_{C}^{\lambda-1} \int_{0}^{\omega} h(s) v_{n}^{\lambda}(s) \mathrm{d} s+\frac{\mu_{n}}{\left\|u_{n}\right\|_{C}} v(t) \quad \text { for } t \in[0, \omega], n \in \mathbb{N},
$$

and, thus, passing the limit for $n \rightarrow+\infty$ and taking into account (4.13) and (4.14), we get

$$
v_{0}(t) \geq h_{0}+\mu_{0} v(t) \quad \text { for } t \in[0, \omega] .
$$

Let us show that $h_{0}+\mu_{0}>0$. Indeed, suppose on the contrary that $h_{0}=0$ and $\mu_{0}=0$. Then, by the hypothesis $p \in \mathcal{V}^{-}(\omega)$, it follows from (4.10) and Remarks 2.3 and 2.4 that

$$
\left|v_{n}(t)\right| \leq \Delta(p)\left(\left\|u_{n}\right\|_{C}^{\lambda-1} \int_{0}^{\omega} h(s) v_{n}^{\lambda}(s) \mathrm{d} s+\frac{\mu_{n}}{\left\|u_{n}\right\|_{C}} \int_{0}^{\omega}|f(s)| \mathrm{d} s\right)
$$

for $t \in[0, \omega], n \in \mathbb{N}$, and, therefore, passing the limit for $n \rightarrow+\infty$ and taking into account (4.13) and (4.14), we obtain

$$
\left|v_{0}(t)\right| \leq \Delta(p)\left(h_{0}+\mu_{0} \int_{0}^{\omega}|f(s)| \mathrm{d} s\right)=0 \quad \text { for } t \in[0, \omega] .
$$

However, this contradicts (4.15). Hence, we have proved that $h_{0}+\mu_{0}>0$, which, together with (4.18) and the positivity of $v$, leads to the condition

$$
v_{0}(t)>0 \text { for } t \in[0, \omega] .
$$

On the other hand, (4.11) yields

$$
\int_{0}^{\omega} h(s) v_{n}^{\lambda}(s) \mathrm{d} s \leq \frac{1}{\left\|u_{n}\right\|_{C}^{\lambda-1}} \int_{0}^{\omega}|p(s)| \mathrm{d} s \quad \text { for } n \in \mathbb{N},
$$

and, therefore, passing the limit for $n \rightarrow+\infty$ and taking into account (4.8) and (4.14), we get

$$
\int_{0}^{\omega} h(s) v_{0}^{\lambda}(s) \mathrm{d} s \leq 0 .
$$

However, in view of (4.19), the latter inequality contradicts (3.1). The obtained contradiction proves that (4.7) holds.

Now we show that the sequence $\left\{\mu_{n}\right\}_{n=1}^{\infty}$ is bounded. Suppose on the contrary that $\sup \left\{\mu_{n}: n \in \mathbb{N}\right\}=+\infty$. Then, we can assume without loss of generality that

$$
\lim _{n \rightarrow+\infty} \mu_{n}=+\infty
$$

Integrating the equation in (1.1) with $\mu=\mu_{n}$ over the interval $[0, \omega]$, we get

$$
0=\int_{0}^{\omega} p(s) u_{n}(s) \mathrm{d} s-\int_{0}^{\omega} h(s) u_{n}^{\lambda}(s) \mathrm{d} s+\mu_{n} \int_{0}^{\omega} f(s) \mathrm{d} s \quad \text { for } n \in \mathbb{N},
$$

which, in view of (3.1) and the positivity of $u_{n}$ and $\mu_{n}$, yields

$$
-\int_{0}^{\omega} f(s) \mathrm{d} s \leq \frac{\left\|u_{n}\right\|_{C}}{\mu_{n}} \int_{0}^{\omega}|p(s)| \mathrm{d} s \quad \text { for } n \in \mathbb{N} .
$$

Taking into account (4.7), (4.20) and passing the limit for $n \rightarrow+\infty$, we obtain $-\int_{0}^{\omega} f(s) \mathrm{d} s \leq 0$, which contradicts the second condition in (3.2). 
Lemma 4.7. Let $p \in \mathcal{V}^{-}(\omega)$ and $z \in A C^{1}([0, \omega])$ be such that

$$
\begin{gathered}
z^{\prime \prime}(t) \leq p(t) z(t) \quad \text { for a. e. } t \in[0, \omega], \quad z(0)=z(\omega), \quad z^{\prime}(0)=z^{\prime}(\omega), \\
\text { meas }\left\{t \in[0, \omega]: z^{\prime \prime}(t)<p(t) z(t)\right\}>0 .
\end{gathered}
$$

Then, $z(t)>0$ for $t \in[0, \omega]$.

Proof. It follows from the hypotheses of the lemma that $z$ is a solution to problem (4.6), where $q(t) \leq 0$ for a. e. $t \in[0, \omega]$ and $q(t) \not \equiv 0$. Therefore, Lemma 4.5 yields $z(t)>0$ for $t \in[0, \omega]$.

Lemma 4.8. Let $\lambda>1, \mu_{0}>0, p \in \mathcal{V}^{-}(\omega),(p, f) \in \mathcal{U}(\omega)$, h satisfy (3.1), and there exist a positive function $\beta \in A C^{1}([0, \omega])$ such that (4.1) with $\mu=\mu_{0}$ and (4.2) hold. Then, for any $\mu \in] 0, \mu_{0}\left[\right.$, there exist functions $\beta_{1}, \beta_{2} \in A C^{1}([0, \omega])$ satisfying conditions (4.3), (4.4), and (4.5).

Proof. Let $\mu \in] 0, \mu_{0}\left[\right.$ be arbitrary and put $\beta_{2}(t):=\frac{\mu}{\mu_{0}} \beta(t)$ for $t \in[0, \omega]$. It follows from (4.1) with $\mu=\mu_{0}$ and (4.2) that $\beta_{2} \in A C^{1}([0, \omega])$ and

$$
\begin{gathered}
\beta_{2}(t)>0 \text { for } t \in[0, \omega] \\
\beta_{2}(0)=\beta_{2}(\omega), \quad \beta_{2}^{\prime}(0)=\beta_{2}^{\prime}(\omega), \\
\beta_{2}^{\prime \prime}(t) \leq p(t) \beta_{2}(t)-\left(\frac{\mu_{0}}{\mu}\right)^{\lambda-1} h(t) \beta_{2}^{\lambda}(t)+\mu f(t) \\
\leq p(t) \beta_{2}(t)-h(t) \beta_{2}^{\lambda}(t)+\mu f(t) \quad \text { for a. e. } t \in[0, \omega],
\end{gathered}
$$

and

$$
\operatorname{meas}\left\{t \in[0, \omega]: \beta_{2}^{\prime \prime}(t)<p(t) \beta_{2}(t)-h(t) \beta_{2}^{\lambda}(t)+\mu f(t)\right\}>0,
$$

because $0<\mu<\mu_{0}$ and $h$ satisfies (3.1). By the hypothesis $(p, f) \in \mathcal{U}(\omega)$ and the condition $\mu>0$, the problem

$$
v^{\prime \prime}=p(t) v+\mu f(t) ; \quad v(0)=v(\omega), v^{\prime}(0)=v^{\prime}(\omega)
$$

has a unique solution $v$ which is positive. In view of (3.1) and (4.23), conditions (4.25) and (4.27) yield

$$
v^{\prime \prime}(t) \geq p(t) v(t)-h(t) v^{\lambda}(t)+\mu f(t) \quad \text { for a. e. } t \in[0, \omega]
$$

and

$$
\left(\beta_{2}(t)-v(t)\right)^{\prime \prime} \leq p(t)\left(\beta_{2}(t)-v(t)\right) \quad \text { for a. e. } t \in[0, \omega]
$$

Therefore, by (4.24), (4.27), and the hypothesis $p \in \mathcal{V}^{-}(\omega)$, we get

$$
v(t) \leq \beta_{2}(t) \text { for } t \in[0, \omega]
$$

Now, by virtue of $(4.24),(4.25),(4.27),(4.28)$, and (4.29), we conclude that the functions $v$ and $\beta$ form a well-ordered pair of lower and upper functions of (1.1) and, thus, problem (1.1) has a solution $\beta_{1}$ such that

$$
v(t) \leq \beta_{1}(t) \leq \beta_{2}(t) \text { for } t \in[0, \omega]
$$

(see, e.g., [3, Chapter I]). Consequently, the functions $\beta_{1}, \beta_{2}$ satisfy conditions (4.4) and (4.5). We finally show that (4.3) holds as well. Indeed, let $z(t):=$ 
$\beta_{2}(t)-\beta_{1}(t)$ for $t \in[0, \omega]$. Since $\beta_{1}$ is a solution to problem (1.1) and $\beta_{2}$ satisfies (4.24), (4.25), and (4.26), we get

$$
\begin{gathered}
z(0)=z(\omega), \quad z^{\prime}(0)=z^{\prime}(\omega) \\
z^{\prime \prime}(t)=p(t) z(t)-h(t)\left(\beta_{2}^{\lambda}(t)-\beta_{1}^{\lambda}(t)\right)-\ell(t) \quad \text { for a. e. } t \in[0, \omega],
\end{gathered}
$$

where $\ell \in L([0, \omega])$ is such that

$$
\ell(t) \geq 0 \quad \text { for a. e. } t \in[0, \omega], \quad \ell(t) \not \equiv 0 .
$$

Therefore, in view of (3.1) and (4.30), the function $z$ satisfies (4.21) and (4.22). Consequently, Lemma 4.7 implies that $\beta_{2}(t)>\beta_{1}(t)$ for $t \in[0, \omega]$, which, together with (4.30) and the positivity of $v$, results in (4.3).

Lemma 4.9. Let $\lambda>1, \mu_{0}>0, p, h, f \in L([0, \omega])$, h satisfy (3.1), and there exist functions $\beta_{1}, \beta_{2} \in A C^{1}([0, \omega])$ such that (4.3), (4.4) with $\mu=\mu_{0}$, and (4.5) hold. Then, there exist $\mu>\mu_{0}$ and a positive function $\beta \in A C^{1}([0, \omega])$ satisfying (4.1) and (4.2).

Proof. It is clear that there exist the numbers $d_{1}>c_{1}>0$ and $d_{2}>c_{2}>0$ such that

$$
c_{1} \leq \beta_{1}(t) \leq d_{1}, \quad c_{2} \leq \beta_{2}(t)-\beta_{1}(t) \leq d_{2} \quad \text { for } t \in[0, \omega] .
$$

Let $\vartheta \in] 0,1[$ be arbitrary. Put

$$
M:=\left\{\left(x_{1}, x_{2}\right) \in \mathbb{R}^{2}: c_{1} \leq x_{1} \leq d_{1}, c_{2} \leq x_{2}-x_{1} \leq d_{2}\right\}
$$

and

$$
\ell\left(x_{1}, x_{2}\right):=\frac{\vartheta x_{1}^{\lambda}+(1-\vartheta) x_{2}^{\lambda}}{\left[\vartheta x_{1}+(1-\vartheta) x_{2}\right]^{\lambda}} \quad \text { for }\left(x_{1}, x_{2}\right) \in M .
$$

Since the function $x \mapsto x^{\lambda}$ is strictly convex on ]0, $+\infty[$, we have

$$
\left[\vartheta x_{1}+(1-\vartheta) x_{2}\right]^{\lambda}<\vartheta x_{1}^{\lambda}+(1-\vartheta) x_{2}^{\lambda} \text { for } 0<x_{1}<x_{2},
$$

which implies that $\ell\left(x_{1}, x_{2}\right)>1$ for $\left(x_{1}, x_{2}\right) \in M$. The function $\ell$ is continuous on the compact set $M$ and, thus, there exists $\varepsilon>1$ such that

$$
\varepsilon^{\lambda-1}\left[\vartheta x_{1}+(1-\vartheta) x_{2}\right]^{\lambda} \leq \vartheta x_{1}^{\lambda}+(1-\vartheta) x_{2}^{\lambda} \quad \text { for }\left(x_{1}, x_{2}\right) \in M .
$$

We now put

$$
\beta(t):=\varepsilon \vartheta \beta_{1}(t)+\varepsilon(1-\vartheta) \beta_{2}(t) \text { for } t \in[0, \omega] .
$$

In view of (4.3) and the conditions $\vartheta \in] 0,1[$ and $\varepsilon>1$, the function $\beta$ is positive and satisfies (4.2). Moreover, from (3.1), (4.4) with $\mu=\mu_{0},(4.31)$, and (4.32), we get

$$
\begin{aligned}
\beta^{\prime \prime}(t) & \leq p(t) \beta(t)-h(t) \varepsilon\left[\vartheta \beta_{1}^{\lambda}(t)+(1-\vartheta) \beta_{2}^{\lambda}(t)\right]+\varepsilon \mu_{0} f(t) \\
& \leq p(t) \beta(t)-h(t) \varepsilon^{\lambda}\left[\vartheta \beta_{1}(t)+(1-\vartheta) \beta_{2}(t)\right]^{\lambda}+\varepsilon \mu_{0} f(t) \\
& =p(t) \beta(t)-h(t) \beta^{\lambda}(t)+\varepsilon \mu_{0} f(t) \quad \text { for a. e. } t \in[0, \omega],
\end{aligned}
$$

i. e., $\beta$ satisfies also (4.1) with $\mu=\varepsilon \mu_{0}>\mu_{0}$. 


\section{Proofs of MAIN RESUlts}

Proof of Theorem 3.1. Conclusion (1) of the theorem follows immediately from [7, Corollary 2.11].

Put

$$
\mathcal{A}:=\{\mu>0: \text { problem }(1.1) \text { has a positive solution }\} .
$$

In view of Lemma 4.3 , it is clear that $\mathcal{A} \neq \emptyset$. Let

$$
\mu_{0}:=\sup \mathcal{A} \text {. }
$$

Then, $\mu_{0}>0$ and Lemma 4.6 implies that $\mu_{0}<+\infty$. Therefore, conclusion (4) of the theorem holds.

We now show that

$$
\mu_{0} \in \mathcal{A} \text {. }
$$

Indeed, let $\left\{\mu_{n}\right\}_{n=1}^{\infty}$ be a sequence of positive numbers such that

$$
\mu_{n} \in \mathcal{A} \quad \text { for } n \in \mathbb{N}, \quad \lim _{n \rightarrow+\infty} \mu_{n}=\mu_{0} .
$$

Moreover, for any $n \in \mathbb{N}$, let $u_{n}$ be a positive solution to problem (1.1) with $\mu=\mu_{n}$. Then, Lemma 4.6 yields (4.7). By the standard arguments used in the proof of a well-posedness of the periodic problem for second-order ODEs, one can show that there exists a subsequence $\left\{u_{n_{k}}\right\}_{k=1}^{\infty}$ of $\left\{u_{n}\right\}_{n=1}^{\infty}$ such that

$$
\lim _{k \rightarrow+\infty} u_{n_{k}}^{(i)}(t)=u_{0}^{(i)}(t) \quad \text { uniformly on }[0, \omega], i=0,1,
$$

where $u_{0} \in A C^{1}([0, \omega])$ is a solution to problem (1.1) with $\mu=\mu_{0}$. All the functions $u_{n_{k}}$ are positive and, thus, it is clear that

$$
u_{0}(t) \geq 0 \text { for } t \in[0, \omega]
$$

By virtue of the hypothesis $(p, f) \in \mathcal{U}(\omega)$, problem (4.16) has a unique solution $v$ which is positive. Since $u_{0}$ is a solution to problem (1.1) with $\mu=\mu_{0}$, by (3.1), (5.3), and (4.16), we get (4.21), where $z(t):=u_{0}(t)-\mu_{0} v(t)$ for $t \in[0, \omega]$. Therefore, the hypothesis $p \in \mathcal{V}^{-}(\omega)$ yields $z(t) \geq 0$ for $t \in[0, \omega]$. Hence, we have

$$
u_{0}(t) \geq \mu_{0} v(t)>0 \quad \text { for } t \in[0, \omega]
$$

and, thus, condition (5.2) holds.

Having a positive solution $u_{0}$ to problem (1.1) with $\mu=\mu_{0}$, it is clear that all the hypotheses of Lemma 4.8 (with $\beta(t):=u_{0}(t)$ ) are fulfilled. Consequently, for any $\mu \in] 0, \mu_{0}\left[,(p, \mu f) \in \mathcal{U}(\omega)\right.$ and there exist functions $\beta_{1}, \beta_{2} \in A C^{1}([0, \omega])$ satisfying (4.3), (4.4), and (4.5). Therefore, conclusion (2) of the theorem follows from Lemma 4.2 .

Since $u_{0}$ is a positive solution to problem (1.1) with $\mu=\mu_{0}$, to prove conclusion ((3)) of the theorem, it is sufficient to show that problem (1.1) with $\mu=\mu_{0}$ has at most one positive solution. Suppose on the contrary that there exists a positive solution to problem (1.1) with $\mu=\mu_{0}$ different from $u_{0}$. Since $\mu_{0}>0,(3.2)$ yields $\left(p, \mu_{0} f\right) \in \mathcal{U}(\omega)$ and, thus, it follows from Lemma 4.1 (with $\beta(t):=u_{0}(t)$ and $\left.\mu:=\mu_{0}\right)$ that problem (1.1) with $\mu=\mu_{0}$ possesses solutions $u_{*}, u^{*}$ such that

$$
u^{*}(t)>u_{*}(t)>0 \text { for } t \in[0, \omega] .
$$


Therefore, Lemma 4.9 (with $\beta_{1}(t):=u_{*}(t)$ and $\beta_{2}(t):=u^{*}(t)$ ) guarantees that there exist $\tilde{\mu}>\mu_{0}$ and a positive function $\beta \in A C^{1}([0, \omega])$ satisfying (4.1) with $\mu=\tilde{\mu}$ and (4.2). Consequently, in view of the hypothesis $(p, f) \in \mathcal{U}(\omega)$ and the positivity of $\tilde{\mu}$, it follows from Lemma 4.1 (with $\mu:=\tilde{\mu}$ ) that problem (1.1) with $\mu=\tilde{\mu}$ has at least one positive solution. However, this implies $\tilde{\mu} \in \mathcal{A}$, which contradicts (5.1).

Proof of Proposition 3.4. By Remark 3.3, it follows from (3.8) that condition (3.2) holds. Let $\mu_{0}$ be the number appearing in the conclusion of Theorem 3.1.

We first show that $\mu_{0}$ satisfies (3.10). Suppose on the contrary that (3.10) does not hold, i.e.,

$$
\mu_{0}<\frac{(\lambda-1)[\Delta(p)]^{-\frac{\lambda}{\lambda-1}}}{\lambda\left[\lambda \int_{0}^{\omega} h(s) \mathrm{d} s\right]^{\frac{1}{\lambda-1}} \int_{0}^{\omega}[f(s)]_{-} \mathrm{d} s} .
$$

Then, it follows from Lemmas 4.3 and 4.2 that problem (1.1) with $\mu=\mu_{0}$ has at least two positive solutions, which contradicts conclusion (3) of Theorem 3.1.

Now we show that $\mu_{0}$ satisfies (3.11). Suppose on the contrary that (3.11) does not hold, i.e.,

$$
\mu_{0} \geq \frac{(\lambda-1)\left[\mathrm{e}^{\frac{\omega}{4} \int_{0}^{\omega}[p(s)]_{+} \mathrm{d} s} \int_{0}^{\omega}[p(s)]_{+} \mathrm{d} s-\int_{0}^{\omega}[p(s)]_{-} \mathrm{d} s\right]^{\frac{\lambda}{\lambda-1}}}{\lambda\left[\lambda \int_{0}^{\omega} h(s) \mathrm{d} s\right]^{\frac{1}{\lambda-1}}\left[\int_{0}^{\omega}[f(s)]_{-} \mathrm{d} s-\mathrm{e}^{\frac{\omega}{4} \int_{0}^{\omega}[p(s)]_{+} \mathrm{d} s} \int_{0}^{\omega}[f(s)]_{+} \mathrm{d} s\right]} .
$$

Then, it follows from Lemma 4.4 that problem (1.1) with $\mu=\mu_{0}$ has no positive solution, which contradicts conclusion (3) of Theorem 3.1.

\section{Model EXAMPLES}

In this section, we consider the model equation (1.3) with $F$ given by the relations in (1.4) in order to demonstrate a possible use of Theorem 3.1.

Let us choose $\omega>0$ and consider the equation

$$
x^{\prime \prime}=\frac{k(\ell-d(t))}{m d(t)} x-\frac{k \ell}{2 m d^{3}(t)} x^{3}-\frac{f_{0}}{m},
$$

where $m, k, \ell, f_{0}>0$ and $\left.d: \mathbb{R} \rightarrow\right] 0,+\infty[$ is a positive $\omega$-periodic function such that $d(t) \not \equiv \ell$ and

$$
\int_{0}^{\omega}\left[\frac{\ell-d(s)}{d(s)}\right]_{-} \mathrm{d} s<\frac{4 m}{\omega k}, \quad \int_{0}^{\omega}\left[\frac{\ell-d(s)}{d(s)}\right]_{+} \mathrm{d} s<\frac{\int_{0}^{\omega}\left[\frac{\ell-d(s)}{d(s)}\right]_{-} \mathrm{d} s}{1-\frac{\omega k}{4 m} \int_{0}^{\omega}\left[\frac{\ell-d(s)}{d(s)}\right]_{-} \mathrm{d} s} .
$$

Observe that equation (6.1) is a Duffing equation with non-constant coefficients and a constant forcing term. By Remarks 3.3 and 3.5, we get

$$
\frac{k(\ell-d(\cdot))}{m d(\cdot)} \in \mathcal{V}^{-}(\omega), \quad\left(\frac{k(\ell-d(\cdot))}{m d(\cdot)},-\frac{1}{m}\right) \in \mathcal{U}(\omega) .
$$

Therefore, assuming that $f_{0}$ is a bifurcation parameter, it follows from Theorem 3.1 that there exists a critical value $f_{0}^{*}>0$ of $f_{0}$ such that, crossing the value $f_{0}^{*}$, a bifurcation of positive $\omega$-periodic solutions to (6.1) occurs. 
As a second example, we consider the equation

$$
x^{\prime \prime}=\frac{k\left(\ell-d_{0}\right)}{m d_{0}} x-\frac{k \ell}{2 m d_{0}^{3}} x^{3}+\frac{A}{m}\left(\sin \frac{2 \pi t}{\omega}-\frac{1}{2}\right),
$$

where $A, \omega>0$ and $m, k, \ell, d_{0}>0$ such that $d_{0}<\ell$ and

$$
\int_{0}^{\omega}\left[\sin \frac{2 \pi s}{\omega}-\frac{1}{2}\right]_{-} \mathrm{d} s>\mathrm{e}^{\frac{\omega^{2} k\left(\ell-d_{0}\right)}{4 m d_{0}}} \int_{0}^{\omega}\left[\sin \frac{2 \pi s}{\omega}-\frac{1}{2}\right]_{+} \mathrm{d} s .
$$

Unlike the first example, equation (6.2) is a Duffing equation with constant coefficients and a sign-changing forcing term. By Remarks 2.2 and 3.5, we get

$$
\frac{k\left(\ell-d_{0}\right)}{m d_{0}} \in \mathcal{V}^{-}(\omega), \quad\left(\frac{k\left(\ell-d_{0}\right)}{m d_{0}}, \frac{g(\cdot)}{m}\right) \in \mathcal{U}(\omega),
$$

where $g(t):=\sin \frac{2 \pi t}{\omega}-\frac{1}{2}$. Therefore, if we consider $A$ as a bifurcation parameter, it follows from Theorem 3.1 that there exists a critical value $A^{*}>0$ of $A$ such that, crossing the value $A^{*}$, a bifurcation of positive $\omega$-periodic solutions to $(6.2)$ occurs.

We finally mention that Proposition 3.4 provides lower and upper estimates of the critical values $f_{0}^{*}$ and $A^{*}$ of bifurcation parameters $f_{0}$ and $A$.

\section{REFERENCES}

[1] A. Cabada, J.Á. Cid and L. López-Somoza, Maximum Principles for the Hill's Equation, Academic Press, London, 2018.

[2] H. Chen and Y. Li, Bifurcation and stability of periodic solutions of Duffing equations, Nonlinearity 21 (2008), 2485-2503.

[3] C. De Coster and P. Habets, Two-Point Boundary Value Problems: Lower and Upper Solutions, Mathematics in Science and Engineering 205, Elsevier Science, Amsterdam, 2006.

[4] C. Fabry, J. Mawhin and M. N. Nkashama, A multiplicity result for periodic solutions of forced nonlinear second order ordinary differential equations, Bull. London Math. Soc. 18 (1986), 173-180.

[5] S. Gaete and R. F. Manásevich, Existence of a pair of periodic solutions of an O.D.E. generalizing a problem in nonlinear elasticity, via variational methods, J. Math. Anal. Appl. 134 (1988), 257-271.

[6] A. Lomtatidze, Theorems on differential inequalities and periodic boundary value problem for second-order ordinary differential equations, Mem. Differ. Equ. Math. Phys. 67 (2016), $1-129$.

[7] A. Lomtatidze and J. Šremr, On a periodic problem for second-order Duffing type equations, Institute of Mathematics, Czech Academy of Sciences, Preprint No. 73-2015 (2015).

[8] R. Ortega, Stability and index of periodic solutions of an equation of Duffing type, Boll. Unione Mat. Ital., Ser. VII. B 3 (1989), 533-546.

[9] J. Šremr, Positive periodic solutions to the forced non-autonomous Duffing equations, Georgian Math. J., to appear.

[10] P. J. Torres, Existence of one-signed periodic solutions of some second-order differential equations via a Krasnoselskii fixed point theorem, J. Differ. Equations 190 (2003), 643-662.

Jiří Šremr, Institute of Mathematics, Faculty of Mechanical Engineering, Brno University of Technology, Technická 2, 61669 Brno, Czech Republic

e-mail: sremr@fme.vutbr.cz 